\title{
Combat Power Emerging Effect of Mathematical Model
}

\author{
Chuanfu Guo ${ }^{1, a}$ and Liang Ma ${ }^{1, b}$ \\ ${ }^{1}$ Department of Surface Ship Command, Dalian Naval Academy, Dalian, 116018, China \\ a285892287@qq.com, bmaliang2014@tom.com
}

\begin{abstract}
Keywords: Combat; Power; Mathematical model
\end{abstract}
\begin{abstract}
In today's world, all countries to speed up the pace of the construction of the fighting, the rapid development of science and technology makes the increasingly fierce competition in the field of military. Combat theory, combat forces, military establishment system as well as a variety of combat system involved in all aspects of the military construction such as the fighting capacity of forging "sharp". The author thought the nature of battle effectiveness factors (material, energy, information) interaction, integration and fusion of the dominant element of the new quality. These new qualitative elements through the arrangement, combination and coordination, evolution showed different effective unit, different functions of combat power unit through integration become integrated operational system of effective system, make the development of nonlinear increase the army battle effectiveness.
\end{abstract}

\section{Combat Effectiveness Emergence Effect Mechanism}

Based on information system of the system operation is the main form of future sea warfare. System to participate in combat all the forces fighting capacity system is the main force. The mechanism of operating system is through the efficient coordination between each operational unit, realize seamless link between each combat power subsystem, in order to achieve the purpose of fighting capacity system function multiplication. For how to achieve effective coordination, many scholars have carried on the thorough discussion, game theory method are the major research methods, the method based on the modeling and simulation of the Agent, the method of distributed interactive simulation (dis), etc., the results of this study for further study of the system operational synergy has played a good role. However, due to the different research starting point and Angle research results from the application level to more study of the collaborative problem, aimed at the problem of more focused on specific goals, plans, such as coordination, rarely from the mechanism level to the coordination problem in-depth discussion. German physicist hawking in the 2 o century proposed the theory of collaborative $7 \mathrm{o} \mathrm{s}$. Synergy theory argues that in an open system, due to the system to maintain a continuous exchange of matter and energy with the outside world, the system itself will be formed to promote each subsystem in the system of the power of synergy, namely "collaborative force". This collaborative force has the positive and negative, negative, it damage in the synergy between each subsystem, system leads to the confusion and chaos system; As timing, to promote synergy, and produce a kind of negative entropy flow, make the system tend to be highly stable and orderly. It is the study of system through internal synergy between subsystems from disorderly to orderly structural transformation mechanism and rules of discipline.

Synergetic is composed of a large number of subsystems in the study of macroscopic behavior in the system, but only from the micro level of parameter cannot understand the macroscopic quantity. Therefore, describes a large number of subsystems of the macro effect of collective motion; need to introduce different from microscopic parameters of macro parameters. Order parameter is introduced to describe the overall system behavior and macro parameter. Order parameter is the product of the collective motion micro subsystem, the cooperation effect of characterization and measurement. Order parameter form reason, not to impose external effect on system, but from within the system. When so many subsystem when the initial state of the system is in chaos, consisting of each subsystem independence movement, chart, there is no relationship, can't form order parameter; And when the system is close to the near point, subsystems, form a cooperative relationship, order parameter form, prompting marks system has entered a new orderly state. Order 
parameter control subsystem, the evolution process of the whole control system. Characterization of order parameter as a system of many subsystems cooperation effect once formed will generate a dominant role and the movement of all subsystems, the existence conditions of each other.

\section{Effective Emergence Effect Model}

Synergy is the soul of system operations, is to organize the implementation of the core of system operations. System power effect each subsystem is not joint can appear immediately, but the role of fighting capacity generation patterns are needed to produce and growth, this is the slow variables, namely the order parameter. It said system orderly structure and type, is the concentrated reflection of subsystem intervention degree of cooperative movement. Power is the power of fighting capacity system operational synergies between subsystems, and also ACTS as a dominant subsystem. Combat power system state, therefore, the spatial and temporal patterns are generated by fighting behavior of the order parameter to treat, and the other subsystems can apply to order parameter. Combat power system as a complex system is supported by multiple combat forces, and each combat power itself is a complex system, the system will show the complex behavior. They are in highly complex ways. Therefore, effective system can be seen as a dynamic system, using collaborative approach to deal with, i.e. it through cooperation between each combat power, emerging new attribute in the form of self-organization.

Combat power system power emerging to meet the following conditions:

Condition 1: combat power system due to many support and guarantee for its combat effectiveness will be improved.

Condition 2: fighting capacity system emerges stronger than a single combat forces combined.

I.e., set up effective system of power $U$, combat power in the system the $i$ combat forces $V_{i}=\left\{v_{i 1}, v_{i 2} \ldots, v_{i l}\right\} i=1,2, \ldots n$ for which the said composition system of combat effectiveness is different; $l=1,2, \ldots m \quad V_{i}^{\prime}=\left\{v_{i 1}^{\prime}, v_{i 2}^{\prime} \ldots, v_{i l}^{\prime}\right\}(i=1,2, \ldots n ; l=1,2, \ldots m)$ Said the first combat forces of order parameter subsystem component), did not take the system operational style of order parameter for the first combat forces due to the action in a separate carries out combat missions, attack the force of traction and the limitation of their own command support capability, if only to combat strength accumulation, there must be Angle between its power, two kinds of strength is not to force the same direction, will produce a internal friction. Under the condition of system operations, through the system of the coupling and the multiplier effect, under the unified effective control coordination, make each combat forces in parallel and in the same direction, therefore $\max \sum_{i=1}^{n} V_{i}^{\prime}=\max \left(V_{1}^{\prime}+V_{2}^{\prime}+\ldots+V_{i}^{\prime}\right)=\left(V_{1}^{\prime}+V_{2}^{\prime}+\ldots+V_{n}^{\prime}\right)=U$

The fighting capacity system emerged stronger than the sum of joint is not a single operation power. Namely

$$
U>\sum_{i=1}^{n} V_{i}^{\prime}
$$

Proof: assume that all combat power is normal operation, if $U \leq \sum_{i=1}^{n} V_{i}^{\prime}$ : because of $U=\sum_{i=1}^{n} V_{i}$

$$
\sum_{i=1}^{n} V_{i}^{\prime}>\sum_{i=1}^{n} V_{i}
$$

Therefore, there must be more than one $V_{i}^{\prime} \geq V_{i}$, is the ith a fighting force less than single combat fighting force, the fighting strength will is outside the scope of fighting capacity system, the contradiction and propositions. So type (1) was established. 
Between various forces in command mode, operational style differences, training mode, etc., so the effective system of internal forces in the collaborative process, there is inevitably friction will not be able to completely merge. In the case of a "resistance", fighting capacity system to overcome the "resistance" and "work", system energy will continue to weaken. "Resistance" and combat forces between the size of the alignment, the structure of the mechanism of gradient, complementary degrees of combat equipment and so on, the direction and effective system to achieve the goal in the opposite direction. Therefore, we need to have control, make the effective system goal toward positive direction development, keep fighting capacity system integrity; the force is effective collaborative force within the system. In the manner and means to establish an effective and continuous communication is an important means of strengthening combat power system stability. The combat power to strengthen the coordination of multiple relations, overcome various difficulties, ensure the normal operation of combat power system. Because each combat power as the power of fighting capacity system elements long formed the regional segmentation, sui generis, differences in command mode, operational mode, so in fighting system inevitably there is friction. The direction of the friction force is always with a single combat forces fighting capacity of ascension in the opposite direction, the size of the resistance and the power factor, the alignment between the system structure of the gradient, complementary weapons and equipment and so on, and effective system of combat effectiveness is proportional to the change, the greater the resistance, the greater the strength change, its direction and effective system of the whole development goals in the opposite direction, can be represented as (type, $\gamma$ is constant) :

$$
f=-\gamma \frac{d U}{d t}
$$

Combat power system under the action of friction, and will gradually reduce, make the system operational loss of meaning; Effective system in order to overcome the friction effect, through the synergy mechanism, coordinated, make mutual fusion between different combat power, reasonable distribution of units fighting capacity gradually improved. So effective system of collaborative force and fighting capacity of ascension in the same direction. Each combat forces fighting capacity convergence, the better consistency, collaborative force is larger.

$$
F=m a
$$

Type, $m$ for system combat power; $a$ is the magnitude and direction of consistency for its convergence.

Fighting capacity system combat power generation mode as order parameter is through self-organization system state to maintain. Since the formation and evolution of the organizational process structure is an internal process. Effective system of combat effectiveness is determined by the combat forces between the synergy. Effective system of each subsystem under certain conditions, by nonlinear interaction can produce coherent effect and synergy, and through the effect to produce an orderly system structure and function. This synergy sports means that the system is the emergence of the new order, in the macro system self-organization phenomena. Joint operations self-organizing system can be expressed as a typical equation

$$
\frac{\partial U}{\partial t}=(F-f) U-k_{1} U^{3}+C
$$

Type (5) may also be the movement of a single combat forces fighting capacity. Fighting capacity system of the state and subject to change with the passage of time, namely the order parameter as a function of time, said. Assumed time determined by the following factors: (1) the order parameter of fighting capacity system; (2) the control parameters, namely combat power system on the synergy force; (3) as the friction of the combat power system. Said system effective movement is nonlinear, is a constant coefficient.

According to type (5), effective system of potential function equation can be obtained

$$
E(U)=\frac{1}{2}(F-f) U^{2}-\frac{1}{4} k_{1} U^{4}
$$


The momentum describes the operating system is the effective system to produce the coupling with double size and potential effectiveness of characterization. If use $F$ and $P$ powerful vector and respectively with the total momentum of the system, the effective system of combat forces in the role of the force generated when the momentum:

$$
\begin{aligned}
& P=m v=F t \\
& F=\frac{d P}{d t} \\
& F d t=d P
\end{aligned}
$$

Type, $F d t$ for system under the impulse of resultant force, it shows that the system under the impulse of resultant force is equal to the total momentum of the delta.

If use $\sum F_{i}$ of the said system powerful vector and, $\sum \frac{d}{d t} P_{i}$ for fighting capacity total momentum of the system, and then

$$
\sum F_{i}=\frac{d}{d t} \sum m_{i} v_{i}=\frac{d}{d t} \sum P_{i}
$$

Due to friction is present in combat power systems, and internal system of combat forces between competition and coexistence relationship must exist. After two or more operations of power system operations $V_{i}^{\prime}=\left\{v_{i 1}^{\prime}, v_{i 2}^{\prime} \ldots, v_{i l}^{\prime}\right\}$, there are multiple combat effectiveness factors of strength Comply with the new order parameter was fighting capacity system. The order parameter can compromise and cooperation with each other, make effective system to produce the self-organizing, but compete with each other between the order parameter, likely order parameter control system of a pattern, other order parameter as auxiliary materials.

Assumptions: (1) the effective system is composed of two forces; (2) the order parameter to improve the rate $U$ of fighting capacity system $q$, rate $b$. Is:

$$
\frac{\partial U}{\partial t}=q(t)-b(t)
$$

Improve the rate $q$ and loss rate $b$ are related to combat effectiveness $U$ :

$$
\begin{aligned}
& q=\alpha U+\beta \\
& b=\lambda U+\delta
\end{aligned}
$$

Type (11), (12) coefficient, $\alpha 、 \lambda$ have nothing to do with $U$, also don't consider the adverse effects $U$ of the changes on the coefficient. Coefficient is determined by the external factors, these factors are constant, there are $\frac{\partial U}{\partial t}=q(t)-b(t)=(\alpha-\lambda) U$

Equation (13) is a separable variable. We can find that, the effective system of combat power exponential type change.

Combat power system under the condition of self-organization may produce the following results:

(1) Competition and coexistence. Two component system combat interactions between combat powers, produce co-exist at the same time there is competition.

$$
\begin{aligned}
& \frac{\partial V_{1}}{\partial t}=\alpha_{1} V_{1}-\beta_{1} V_{1}^{2} \\
& \frac{\partial V_{2}}{\partial t}=\alpha_{2} V_{2}-\beta_{2} V_{2}^{2}
\end{aligned}
$$

Type, $V_{1}, V_{2}$ respectively two forces fighting capacity; $\alpha_{1}, \alpha_{2}$ respectively, fighting capacity 
variation and the relationship between the combat effectiveness; For $\beta_{1} V_{1}^{2}, \beta_{2} V_{2}^{2}$ effective change is non-linear. Make $\frac{\partial V_{1}}{\partial t}=\frac{\partial V_{2}}{\partial t}=0$ available, steady $V_{1}, V_{2}$ state equation, the solution of this equation can get steady $V_{1}^{0}, V_{2}^{0}$ state solution,. Can be seen from the above analysis, the effective system of competition and coexistence is appeared at the same time.

(2) Symbiosis. Efficient coordination between combat powers, its purpose is to improve the combat effectiveness of combat power system. Therefore, at this time a combat forces fighting strength increase rate will fight with another fighting strength as the condition, it is

$$
\begin{aligned}
& \frac{\partial V_{1}}{\partial t}=\left(\alpha_{1}+\beta_{1} V_{2}\right) V_{1}-\delta_{1} V_{1} \\
& \frac{\partial V_{2}}{\partial t}=\left(\alpha_{2}+\beta_{2} V_{1}\right) V_{2}-\delta_{2} V_{2} \\
& \frac{\partial V_{1}}{\partial t}=\left(\alpha_{1}+\beta_{1} V_{2}\right) V_{1}-\delta_{1} V_{1}=0 \\
& \frac{\partial V_{2}}{\partial t}=\left(\alpha_{2}+\beta_{2} V_{1}\right) V_{2}-\delta_{2} V_{2}=0
\end{aligned}
$$

Solutions of the equations, the solution of the two groups. Group 1: $V_{1}=V_{2}$ no meaning; Solution: 2 groups. $\left\{\begin{array}{l}\alpha_{1}+\beta_{1} V_{2}-V_{1}=0 \\ \alpha_{2}+\beta_{2} V_{1}-V_{2}=0\end{array}\right.$ Through the analysis on the stability of group 2 solution, in and don't to 0 (that is, indicate the presence of a "joint") in the initial conditions, the joint combat effectiveness of the two forces will increase exponentially, this is reflected the system operational synergies. Therefore draw the conclusion: the stronger the fighting capacity system of the combat forces fighting capacity, in fighting system effective promotion potential, the greater and growing exponentially.

\section{Conclusions}

Above all, fighting capacity in the system each fighting capacity between subsystems, due to the command mode, operational mode, training mode, and the difference of weapons and equipment, inevitably produce friction. The direction of the friction force always and fighting capacity system effectiveness of ascension in the opposite direction, size and the alignment between the combat power, the gradient of the command structure, weapons and equipment of complementary degrees and so on. Combat power system under the action of friction, fighting capacity will be reduced; The more effective system of combat forces fighting capacity, form the system of operational effectiveness after promotion potential, the greater the and is not a simple linear relationship, but is growing exponentially. This is the "joint" will be "1+1>2" effect.

\section{References}

[1] Liwei L, Rongshuang F. Simulated annealing algorithm in solving frequency assignment problem[C]//Advanced Computer Theory and Engineering (ICACTE), 2010 3rd International Conference on. IEEE, 2010, 1: V1-361-V1-364.

[2] Castelino D J, Hurley S, Stephens N M. A tabu search algorithm for frequency assignment [J]. Annals of Operations Research, 1996, 63(2): 301-319.

[3] Alabau M, Idoumghar L, Schott R. New hybrid genetic algorithms for the frequency assignment problem [J]. Broadcasting, IEEE Transactions on, 2002, 48(1): 27-34. 
[4] Radiom, S., Aliakbarian, H., etc., A Simple Real-Coded Compact Genetic Algorithm and its Application to Antenna Optimization, Microwave conference, APMC 2007.

[5] Francisco Herrera and Manuel Lozano. Gradual Distributed Real-Coded Genetic Algorithms [J]. IEEE transactions on evolutionary computation, 2000,4(1):43-62.

[6] Díógenes Marcano and Filinto Durán. Synthesis of [7] Antenna Arrays Using Genetic Algorithms [J]. IEEE Antennas and Propagation Magazine, 42(3):12-20.

[7] Wen-Pin Liao, Fu-Lai Chu. Array pattern synthesis with null steering using genetic algorithm by controlling only the current amplitudes [J]. INT. J. ELECTRONICS, 1999, 86(4):445-457.

[8] Wen-Pin Liao, Fu-Lai Chu Application of Genetic Algorithms to Phase-Only Null Steering of Linear Arrays [J] Electromagnetics, 1997, 17:171-183.

[9] Fred Aust in, Michael lew is. Automated Maneuvering Decision for Air-to-Air Combat[R].AIAA-87-2393:659-669 